\title{
Simulations of the PEP-II Transverse Coupled-bunch Feedback System ${ }^{*}$
}

\author{
J. M. Byrd \\ Lawrence Berkeley Laboratory \\ Berkeley, CA 94720 USA
}

\begin{abstract}
We report the results of a computer simulation of the PEPII transverse feedback (TFB) system including single particle transverse beam dynamics and measured RF cavity higher order modes. The simulation addresses issues such as required FB power and gain, noise, bunch offsets, and varying initial bunch conditions. We also summarize a simulation of a proposed mode of operation of the feedback system where selected individual bunches are driven to large amplitudes in order to trim their current.
\end{abstract}

\section{INTRODUCTION}

Coupled \pm bunch instabilities pose one of the problems in operation of high \pm current, multibunch storage rings such as PEP \pm II, an asymmetric electron \pm positron collider with energies of 3.109 and $9 \mathrm{GeV}$. Electromagnetic wakes excited in vacuum chamber components such as RF cavities couple the oscillations of successive bunches which can lead to exponentially growing oscillations. Because transverse oscillations especially degrade the performance of a collider, a transverse feedback (TFB) system is being designed to damp the oscillations[1]. To help guide the feedback design process and to further understanding of multibunch phenomena, a computer simulation of the oscillations and the corresponding feedback systems has been developed. Instabilities are generally of more concern for the PEP \pm II low energy ring and thus this paper treats only that case although similar studies have been conducted for the PEP \pm II high energy ring.

The proposed TFB system is a so \pm called bunch \pm by \pm bunch system which works independently on each bunch. It is very similar to one now in use at the Advanced Light Source (ALS). Implicit in the requirement of acting on each bunch is that the minimum bandwidth of the system be greater than half the bunch frequency, which for $\mathrm{PEP} \pm \mathrm{II}$ is $238 \mathrm{MHz}$. Because the major expense in these systems are the broadband power amplißers, one the most important issues in the simulation is to determine if the speci®ed power is adequate to damp the beam under a variety of conditions.

The ability of the TFB to control individual bunches has led to a proposal to use the system as a means of trimming or eliminating individual bunches without affecting other bunches by by driving them to large transverse amplitudes where they would encounter aperture restrictions. One issue which arose from previous experience with the ALS system is the nonlinearity of the betatron motion causing decoherence and effectively limiting the amplitude to which the bunch could be driven. The last section of this paper brie-y describes a multiparticle/bunch simulation

\footnotetext{
*This work was supported by the Director, Of®ce of Energy Research, Of®ce of High Energy and Nuclear Physics, High Energy Physics Division, of the U.S. Department of Energy under contract No. DE-AC03-76SF00098.
}

\begin{tabular}{clc}
\hline \hline Symbol & Description & LER Value \\
\hline$E$ & Beam energy $(\mathrm{GeV})$ & 3.109 \\
$C$ & Circumference $(\mathrm{m})$ & 2199.318 \\
$f_{r f}$ & RF Freq. & $476 \mathrm{MHz}$ \\
$N$ & No. of bunches & 1658 \\
$I_{0}$ & Beam current $(\mathrm{A})$ & 2.14 \\
$N_{r f}$ & No. of RF cells & 6 \\
$Q_{x, y}$ & x,y $\beta \pm$ tron tunes & $32.28,35.18$ \\
$\left\langle\beta_{x, y}\right\rangle$ & aver. $\beta_{x, y}(m)$ & $10.84,9.95$ \\
\hline \hline
\end{tabular}

Table I

PEP-II LER parameters used in the simulations.

including nonlinear effects in both planes which addresses the issue of decoherence with positive feedback gain.

\section{BEAM DYNAMICS AND WAKE VOLTAGES}

The beam dynamics in this study are simulated using common tracking techniques at a single point in the ring, where difference equations are used to describe the discrete time evolution of the beam oscillations and wake voltages[2], [3]. This approximation is valid for all of the effects we wish to study, especially since most of the wake voltages are localized at the RF cavities. One notable exception is the distributed wake of the resistive wall impedance, which results from the skin effect on the inner vacuum chamber wall. The local approximation for the resistive wall wake is valid when the corresponding growth or damping rate is slow compared to the betatron oscillation period. This condition is true for all cases studied here. Tracking is done in both transverse directions, which we generically label $x$. The turn \pm by \pm turn difference equations for $x$ and $x$ for a linear lattice are given by

$$
x_{i+1}=\cos 2 \pi Q_{x} x_{i}+\beta_{x} \sin 2 \pi Q_{x} x_{i}^{\prime}\left(1-2 \lambda_{x}\right)
$$

$x_{i+1}^{\prime}=-\frac{1}{\beta_{x}} \sin 2 \pi Q_{x} x_{i}+\cos 2 \pi Q_{x} x_{i}^{\prime}\left(1-2 \lambda_{x}\right)+\frac{\Re\left(\tilde{V}_{w, i+1}\right)}{E}$

where the tracking is assumed to be at a symmetry point with $\beta^{\prime}=0$ and zero dispersion. The tunes and $\beta$ functions are assumed to be constant as a function of amplitude and energy. Amplitude \pm dependent tunes generally lower the effective coupled \pm bunch growth rates but in this paper we are restricting ourselves to a worst case scenario with no nonlinearities. The effects of nonlinearities are discussed at the end of this paper with regard to driven motion. Effects of energy oscillations are ignored here. The damping term, $\lambda_{x}$ nominally represents 
the radiation damping but can be adjusted to approximate coherent damping effects such as head \pm tail damping and decoherence which the rigid \pm bunch simulation cannot model.

The transverse wake voltage from dipole HOMs is found by summing the contributions from all previous bunch passages. Tracking of the wake voltage is done by treating it as a complex phasor, $\tilde{V}_{w}$. The difference equation for $\tilde{V}_{w}$ is given by

$$
\tilde{V}_{w, i+1}=\tilde{V}_{w, i} e^{\left(j \omega_{r}+\frac{\omega_{r}}{2 Q}\right) \Delta t}-j 2 k_{\perp} q x_{i+1}
$$

where $k_{\perp}$ is the transverse loss parameter, $\omega_{r}$ is the angular resonant frequency, and $x$ is the transverse offset at the location of the HOM. The wake voltage is out of phase with the motion and thus does not in-uence the charge which generated it.

As mentioned above, the resistive wall wake voltage is the only non \pm localized wake of concern. The approximate localized wake voltage for a round pipe is given by

$$
V_{w, r w}(t)=q x \frac{l c}{\pi b^{3}} \sqrt{\frac{\mu_{0} \rho}{\pi}} \frac{1}{\sqrt{t}}
$$

where $l$ is the length of the vacuum chamber (usually the ring circumference), $\rho$ the material resistivity, and $b$ the chamber radius. Because the inverse square root time dependence of the wake voltage, it is not possible to use the computational trick in Eq. 3 to advance the wake voltage each discrete time step. The voltage for bunch passage must be remembered and individually recalculated each iteration. Since it is not practical to store all previous wake voltages, we use simply cut off the wake voltage after several turns. The number of turns necessary to adequately simulate the wake voltage was determined empirically by increasing the value until no further change in the observed growth rate was seen, which also was resulted in a growth rate in agreement with the calculated growth rate. The value used was either 4 or 5 turns.

\section{A. Feedback Systems}

The TFB system measures a transverse position error and produces the appropriate angular kick to correct the error which is applied to the beam on a subsequent turn. Because the tracking in this simulation is done at only a single point in the ring, we are currently using a linear combination of the transverse position measured on the two previous turns to calculate the correct kick given by

$$
V_{f b}=G_{f b} E\left(\frac{x}{\tan \phi_{x} \beta_{x}}-\frac{x_{-1}}{\sin \phi_{x} \beta_{x}}\right)
$$

where $G_{f b}$ is the TFB gain and usually ranges from $0.03 \pm 0.3$, $x$ and $x_{-1}$ are the positions on the current and previous turns. The FB voltage limits at the maximum ampli®er output, which we take here as $2.2 \mathrm{kV}$.

DC beam offsets at the FB pickups must be reduced by the TFB to maintain the dynamic range of the system. Because the simulation does not explicitly model a ßlter, beam offsets are simulated by simply assuming that a $® 1$ ter reduces the offset by a factor of 10 , which has been shown to be easily achieved.

\begin{tabular}{clc}
\hline \hline Freq $(\mathrm{MHz})$ & $R_{\perp}(k \Omega / m)$ & $\mathrm{Q}$ \\
\hline 1674 & 0.385 & 2134 \\
1435 & 0.342 & 3955 \\
1311 & 0.080142 & 498 \\
1757 & 0.027283 & 7129 \\
1588 & 0.0121 & 178 \\
1203 & 0.0103 & 1588 \\
\hline \hline
\end{tabular}

Table II

Strongest PEP-II RF cavity dipole HOM parameters.

\section{RESULTS}

\section{A. Multibunch Damping}

For the injection scheme of PEP-II, a single bunch out of 1658 is injected with $1 / 5$ the the total bunch charge. For $5 \mathrm{~mm}$ maximum offset of the injected charge, the centroid offset is $1 \mathrm{~mm}$. The dipole HOMs used in the simulation are listed in Table II and the resistive wall impedance is $Z_{r w}=1.53 / \sqrt{n} M \Omega / m$, where $n$ is the frequency scaled by the orbit frequency. In addition, a $10 \%$ bunch \pm bunch coupling through the TFB system was used to simulate its $\circledR$ nite bandwidth.

The result of an injection transient is shown in Figure 1. Figure $1 \mathrm{~b}$ shows an expanded view of the other bunches excited by the injected bunch. Figure 1c plots the amplitude of the voltage kick. For most of the transient, ampli®er is saturated at its maximum value at the injected bunch but operates proportionally for all other bunches. As a result, the damping of the injected bunch is linear in time until the ampli®er comes out of saturation where it becomes exponential. The injected bunch is well \pm damped before the next injection. The steady \pm state offset of the damped beam is discussed above and results in a steady \pm state kick from the ampli®er which consumes about half of the available voltage.

\section{B. Single Bunch Kickout}

To test whether a single bunch could be driven to large amplitudes by the TFB, we tested the simulation with only a single bunch but using 500-1000 particles/bunch. The transverse distribution was initialized to a Gaussian and a random Gaussian deviate is added to Eqns. 1 and 2 according to ref. [2].

The tune spread resulting from the nonlinearity was included in the tracking in a quasi \pm linear manner by implicitly making the betatron tunes a function of amplitude in the form

$$
Q_{x}(\hat{x}, \hat{y})=Q_{x 0}+k_{x} \hat{x}^{2}+k_{x y} \hat{y}^{2}
$$

and

$$
Q_{y}(\hat{x}, \hat{y})=Q_{y 0}+k_{y} \hat{y}^{2}+k_{y x} \hat{x}^{2}
$$

where $\hat{x}^{2}=x^{2}+\left(\beta x^{\prime}\right)^{2}$. The coef®cients $k_{x}, k_{x y}$, and $k_{y}$ are determined from the actual PEP-II lattice[4]. This approach allows us to study the effects of the tune spread without including the effects of the entire lattice and ignores nonlinear dynamics issues.

Particles were considered lost if they exceeded amplitudes of $>10 \sigma$ in either plane. In the simulation, the FB gain was reversed and increase to a large value to destabilize the motion in 


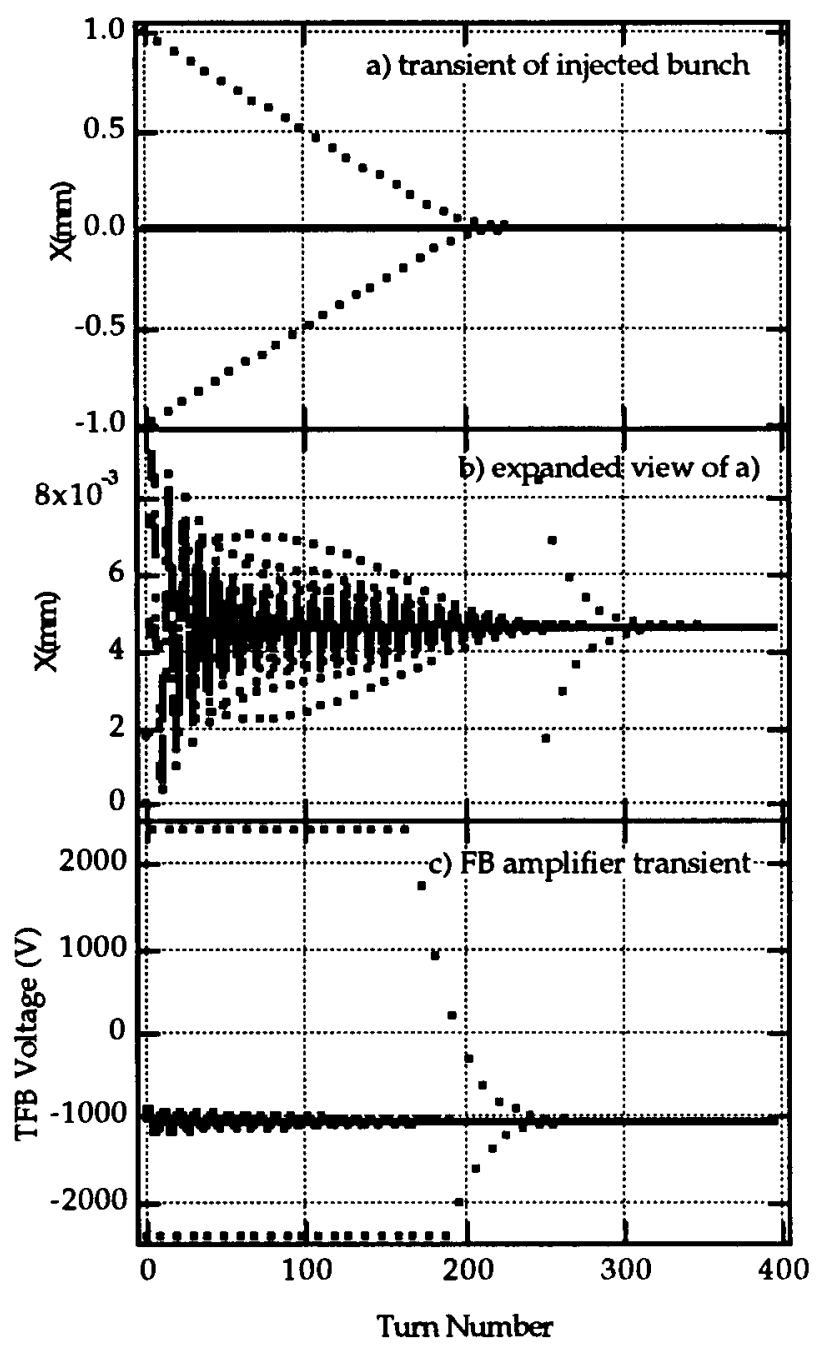

Figure. 1. Transient response of beam and TFB at injection. a) Position vs. turn for $1 \mathrm{~mm}$ injected bunch. b) Expanded view of a) showing transient of stored bunches. c) Response of TFB showing saturation of injected bunch and DC response from offset.

the desired plane, while the FB gain remained constant in the other plane. We found that it was possible to drive the bunch vertically and cause controlled beam loss but very difßcult to drive a bunch horizontally with the available voltage. This occurs because the larger horizontal beam size allows a larger betatron tune spread than in the vertical plane. The larger tune spread across the bunch causes individual particles to dephase or decohere faster, effectively damping the centroid motion. Because the TFB operates on the centroid position of the bunch, the gain effectively vanishes. The situation is illustrated in Figure 2 which shows the horizontal phase space distribution at 4 stages between 0 and 1600 turns. About $50 \%$ of the beam has exceeded an amplitude of $10 \sigma$ after 1600 turns. The vertical plane shows very little decoherence before it is lost.

\section{CONCLUSIONS}

The specißcations of PEP \pm II TFB system are adequate for damping the expected transverse instabilities. Trimming of in-
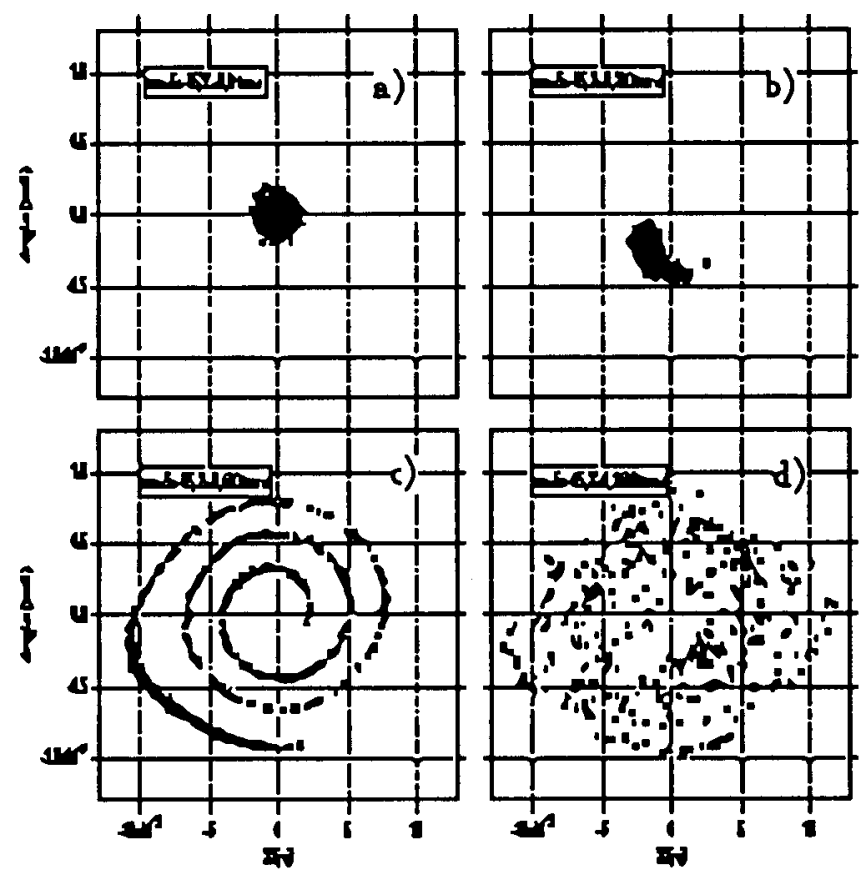

Figure. 2. Horizontal phase space distribution (angle vs. position) at 4 stages following initiation of single bunch kickout. After 1600 turns $50 \%$ of the beam has exceeded an amplitude of $10 \sigma$ but there is very little centroid motion.

dividual bunches is feasible if driven in the vertical direction.

\section{References}

[1] W. Barry, et. al., Design of the PEP-II Transverse Coupled \pm Bunch Feedback System, these proceedings.

[2] R. Siemann, Computer Models of Instabilities in Electron Storage Rings, in The Physics of Particle Accelerators, AIP Conf. Proc. 127, 431 (1983).

[3] K. Thompson, Transverse and Longitudinal Coupled Bunch Instabilities in Trains of Closely Spaced Bunches, PAC 1989 (1989).

[4] E. Forest, et. al., Sources of amplitude \pm dependent tune shift in the PEP \pm II design and their compensation with octupoles, EPAC 1994, May 1994. 